\title{
Spatial Distribution of Low- and High-Voltage-Activated Calcium Currents in Neurons of the Deep Cerebellar Nuclei
}

\author{
Volker Gauck, ${ }^{2}$ Michael Thomann, ${ }^{1}$ Dieter Jaeger, ${ }^{2}$ and Alexander Borst ${ }^{1}$ \\ ${ }^{1}$ Friedrich-Miescher-Laboratory of the Max-Planck-Society, 72076 Tuebingen, Germany, and 2Department of Biology, \\ Emory University, Atlanta, Georgia 30322
}

The spatial distribution of low-voltage-activated (LVA) and highvoltage-activated (HVA) barium currents was investigated in neurons of the deep cerebellar nuclei (DCN) by combining barium imaging with voltage clamp. The current-induced fluorescence signal $(\Delta F / F)$ of the HVA current was five times higher then the LVA-induced signal at the soma, but both signals were approximately equal in size in distant dendrites. This positiondependent shift of $\Delta F / F$ indicates a non-uniform distribution of the underlying calcium channels. The higher weight of the LVA signal in the dendrites suggests that the LVA might be of particular relevance for the dendritic integration of synaptic inputs.

Key words: calcium imaging; low-voltage-activated calcium current; high-voltage-activated calcium current; dendritic integration; subcellular compartmentalization; cerebellar nuclei
Many properties of nerve cells are known to depend on the regulation of intracellular calcium levels. In the present study we investigated neurons of the deep cerebellar nuclei (DCN) that represent the vast majority of the cerebellar output neurons. Several studies indicate the importance that intracellular calcium has for these neurons. The intracellular calcium level of DCN neurons is likely to determine the long-term synaptic plasticity at inhibitory synapses that they receive from Purkinje cells of the cerebellar cortex (Aizenman et al., 1998). These changes correspond to those that have been reported for excitatory synapses in other neuron types (Artola and Singer, 1993). Furthermore, imaging experiments indicated that the somatic calcium level is determined to a large extent by the spiking activity of DCN neurons (Muri and Knopfel, 1994). This calcium level in turn has been suggested to regulate the overall excitability of DCN neurons (Aizenman and Linden, 2000). To understand the potential function of calcium for the properties of DCN neurons, their spatial distribution might be critical. An increasing number of studies point out how significant the spatial distribution of active ionic conductances is for the processing of synaptic inputs and for intracellular signaling (Magee, 1999a). A gradient in the density of $I_{h}$ channels in CA1 pyramidal neurons, for example, has been suggested to compensate for the filter effects that purely passive dendrites would impose on synaptic inputs (Magee, 1999b). Another example is an increasing density of A-type potassium channels toward dendritic tips of CA1 pyramidal neurons that regulate the backpropagation of action potentials (Johnston et al., 2000).

Received March 14, 2001; revised May 9, 2001; accepted May 15, 2001

This work was supported by Research Fellowship GA-627-2 of the Deutsche Forschungsgemeinschaft to V.G., by European Union Biotech Grant PL970182 to A.B. and M.T., and by Grant R29 MH57256 of the National Institute of Mental Health to D.J.

V.G. and M.T. contributed equally to this work.

Correspondence should be addressed to Volker Gauck, University of Tuebingen, Department of Neurology/Cognitive Neurology, Auf der Morgenstelle 15, 72076 Tuebingen, Germany. E-mail: volker.gauck@uni-tuebingen.de.

V. Gauck's present address: Department of Cognitive Neurology, University of Tuebingen, 72076 Tuebingen, Germany.

A. Borst's present address: ESPM-Division of Insect Biology, University of California, Berkeley, CA 94720-3112.

Copyright (C) 2001 Society for Neuroscience $\quad 0270-6474 / 01 / 210001-\bullet \$ 15.00 / 0$
In the present study we used imaging and voltage clamp to examine the spatial distribution of low-voltage-activated (LVA) and high-voltage-activated (HVA) calcium currents in DCN neurons. We found that the relative strength of the low-voltageactivated calcium current increases significantly toward more distal dendrites.

\section{MATERIALS AND METHODS}

Electrophysiology. Whole-cell patch recordings of DCN neurons were obtained with an Axoclamp 2A amplifier in $300 \mu \mathrm{m}$ sagittal cerebellar slices from 11 - to 17 -d-old rats at room temperature $\left(\sim 22^{\circ} \mathrm{C}\right)$. Large and medium-sized neurons from the lateral and intermediate DCN were recorded. Slices were kept at $32^{\circ} \mathrm{C}$ in a solution perfused with carbogen $\left(95 \% \mathrm{O}_{2}, 5 \% \mathrm{CO}_{2}\right.$ ) containing (in $\mathrm{mm}$ ): $\mathrm{NaCl} 125, \mathrm{KCl} \mathrm{3}, \mathrm{MgCl}_{2} 1$, $\mathrm{CaCl}_{2} 2$, glucose $10, \mathrm{NaH}_{2} \mathrm{PO}_{4} 1.25, \mathrm{NaHCO}_{3} 25$. The recording solution was bubbled with oxygen. Its composition was (in mM): $\mathrm{NaCl} 130, \mathrm{KCl} 3$, $\mathrm{MgCl}_{2} 2, \mathrm{CaCl}_{2}$ 2, glucose 10, HEPES 10. Calcium was substituted with barium to prevent calcium-dependent potassium currents from being activated. Voltage clamp was used to measure barium currents after blockage of sodium, potassium, and $\mathrm{I}_{\mathrm{h}}$ channels by adding the following substances to the recording solution (final concentrations in MM): TTX 0.0005, TEA 8, 4-AP 2, CsCl 2. Recording electrodes were filled with (in $\mathrm{mm}$ ): $\mathrm{NaCl} 10$, K-gluconate 134, EGTA 0.2, HEPES 10, Mg-ATP 4, Na-GTP 0.3, phosphocreatine 10. The electrode resistance ranged between 3 and $8 \mathrm{M} \Omega$ and was bridge balanced. After a whole-cell configuration was established, electrodes had a resistance between 3 to $5 \mathrm{M} \Omega$ that was not compensated. All voltages were corrected by the subtraction of a $10 \mathrm{mV}$ junction potential. Data were sampled with $5 \mathrm{kHz}$. The average input resistance was $674 \pm 33 \mathrm{M} \Omega($ mean $\pm \mathrm{SD})(n=31)$ when

This article is published in The Journal of Neuroscience, Rapid Communications Section, which publishes brief, peerreviewed papers online, not in print. Rapid Communications are posted online approximately one month earlier than they would appear if printed. They are listed in the Table of Contents of the next open issue of JNeurosci. Cite this article as: JNeurosci, 2001, 21:RC158 (1-4). The publication date is the date of posting online at www.jneurosci.org.

http://www.jneurosci.org/cgi/content/full/5463 
measured under voltage clamp by stepping from -90 to $-100 \mathrm{mV}$ in a solution containing TTX, 4-AP, TEA, and Cs.

Imaging. Intracellular barium was optically measured with the dye calcium green-1 (29 $\mu \mathrm{M}$, intracellular) on an upright microscope (Zeiss, Axioskop). Cells were viewed using IR-DIC optics and a video camera (Hamamatsu, C2400). After a whole-cell configuration was established, calcium green-1 was allowed to diff use for 15-20 min into the neuron before the recording was started. Pictures $(256 \times 256$ pixels $)$ were taken at a rate of $13 \mathrm{~Hz}$ with a charge-coupled device (CCD) camera (Photometrics, PXL) using a Zeiss $63 \times$ water immersion lens. The CCD camera was operated in frame transfer mode. The barium signal $(\Delta F / F)$ was defined as the fluorescence change $(\Delta F)$ that was induced by a voltage step (stimulus) under voltage clamp divided by the prestimulus fluorescence $(F)$. The prestimulus fluorescence $F$ was not corrected for the background fluorescence of the surrounding tissue. Our signal $(\Delta F / F)$ consequently underestimates rather than overestimates the actual barium influx. Absolute barium concentrations were not measured, and therefore no statements about the current densities are possible. Instead, we compared the strength of the LVA current-induced $\Delta F / F$ and the HVA current-induced $\Delta F / F$ for several dendritic positions. This allowed us to evaluate the relative weight between the LVA and HVA currents as a function of the distance from the soma.

\section{RESULTS}

\section{Voltage dependence of LVA and HVA currents}

In a first set of experiments, the voltage dependence of barium currents in DCN neurons was characterized with whole-cell recordings. Voltage steps from -90 to $-40 \mathrm{mV}$ or below activated exclusively a transient barium current (Fig. 1A). This LVA current is similar to T-type currents that have been described in other neuron types (Fox et al., 1987; Coulter et al., 1989; Huguenard, 1996). The peak of the inward current was taken as a measure for the LVA current. The LVA current reached its maximal value at $-30 \mathrm{mV}$. The inactivation of the LVA was measured by stepping from holding potentials between -90 and $-50 \mathrm{mV}$ to a test potential of $-40 \mathrm{mV}$ (data not shown). The inactivation was almost totally removed at $-85 \mathrm{mV}$, and it was complete at $-50 \mathrm{mV}$ (Fig. $1 C$ ). The half-maximal value was between -65 and $-60 \mathrm{mV}$.

Voltage steps from $-50 \mathrm{mV}$ to more depolarized voltages activated a persistent inward current (Fig. 1B). The average inward current during the last $0.1 \mathrm{sec}$ of a $0.5 \mathrm{sec}$ voltage step was taken as a measure for the HVA current. The channel types that contribute to the HVA current in DCN neurons have not been identified. The inward current that we measured during the HVA protocol showed a slow transient decrease for potentials more positive then $-30 \mathrm{mV}$. This indicates the presence of $\mathrm{N}$-type calcium channels that are known to inactivate in a voltagedependent manner (Fox et al., 1987). A large component of the HVA current, however, was noninactivating and therefore likely to be composed of L-, P-, or R-type currents. The HVA current reached its maximal value at $-20 \mathrm{mV}$ and its half-maximal value between -30 and $-25 \mathrm{mV}$ (Fig. $1 C$ ). The relative currents in Figure $1 C$ correspond to $617 \pm 43 \mathrm{pA}$ (mean $\pm \mathrm{SD}) \mathrm{HVA}(-45$ to $-20 \mathrm{mV}$ ), $832 \pm 34 \mathrm{pA}$ (mean $\pm \mathrm{SD}$ ) LVA (activation: -90 to $-30 \mathrm{mV}$ ), and $340 \pm 41 \mathrm{pA}$ (mean $\pm \mathrm{SD}$ ) LVA (inactivation: -90 to $-45 \mathrm{mV})$.

The voltage dependence of the LVA and HVA currents in Figure $1 C$ is unlikely to match exactly the voltage dependence of the corresponding calcium channels for several reasons. Raman et al. (2000) described recently a persistent voltage-insensitive mixed cation current that had a reversal potential of approximately $-34 \mathrm{mV}$ and was insensitive to TTX, TEA, and 4-AP. This current was not blocked in our experiments, and consequently the reversal potential of the barium current could not be determined accurately. Therefore we decided to plot current
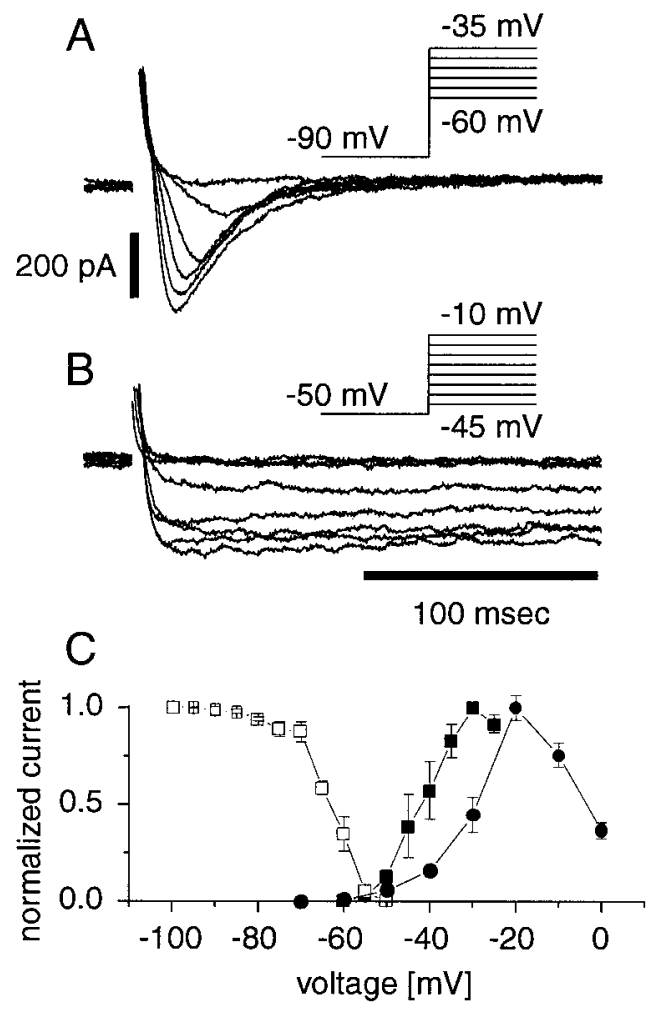

Figure 1. Voltage dependence of LVA and HVA currents. A, LVA current traces of a DCN neuron, activated by voltage steps from $-90 \mathrm{mV}$ to potentials between -60 and $-35 \mathrm{mV}$. B, HVA current traces from the same neuron as in $A$, activated by voltage steps from $-50 \mathrm{mV}$ to potentials between -45 and $-10 \mathrm{mV}$. $C$, Voltage dependence of the LVA inactivation $(\square, n=2)$, the LVA activation $(\square, n=5)$ and the HVA activation $(\boldsymbol{\bullet}, n=15)$. Currents were normalized to maximal values but not corrected for the changing driving force. For stimuli that activated both currents, i.e., steps from -90 to $-30 \mathrm{mV}$, the LVA value was calculated as the peak current minus the persistent current during the last $100 \mathrm{msec}$ of stimulation.

instead of conductance in Figure $1 C$. Furthermore, the electrode series resistance was not compensated (see Materials and Methods); therefore, the current-voltage curves of Figure $1 C$ might be shifted by up to $-4 \mathrm{mV}$ with respect to their true value. The voltage steps that we used in the imaging experiments elicited currents below $400 \mathrm{pA}$. Therefore, the voltage error from an uncompensated series resistance was below $2 \mathrm{mV}$. It is important to note that an uncompensated series resistance does not impair the conclusions of the imaging experiments because somatic and dendritic locations are affected in the same way by the resulting voltage deviation. Despite these limitations, however, Figure 1 demonstrates that the LVA and HVA currents were activated separately. This was crucial for the barium imaging experiments. Stepping from $-90 \mathrm{mV}$ to potentials of $-40 \mathrm{mV}$ or below activated exclusively the LVA current, and stepping from -50 or -45 to $-10 \mathrm{mV}$ activated only the HVA current.

\section{Relative strength of LVA and HVA currents}

To study the spatial distribution of the LVA and HVA currents we measured the barium signal $(\Delta F / F)$ at several distances from the soma. In the experiment of Figure 2, four different locations were evaluated: the soma $(0 \mu \mathrm{m})$, a proximal dendrite $(0-20 \mu \mathrm{m})$, an intermediate dendrite $(20-60 \mu \mathrm{m})$, and a distal dendrite (60-120 $\mu \mathrm{m}$ ) (Fig. 2A,B). The first voltage command was a step from -90 to $-45 \mathrm{mV}$ to activate and thereupon inactivate the 

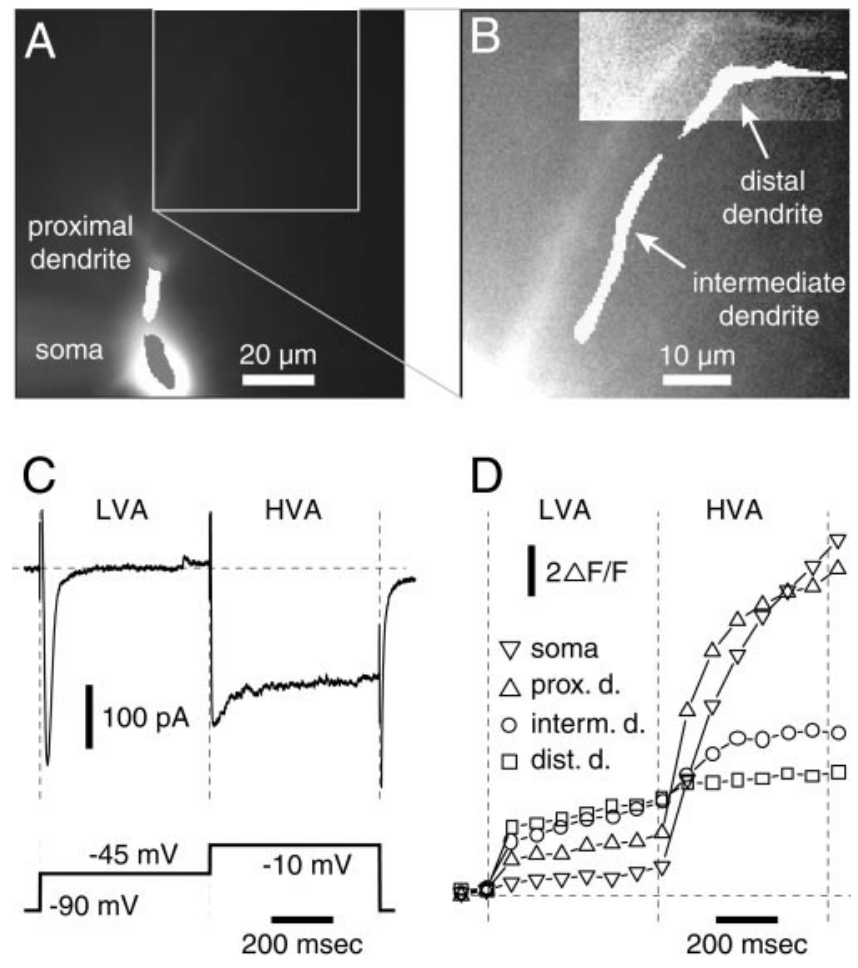

Figure 2. Barium imaging of a DCN neuron under voltage clamp. $A$, Raw fluorescence image showing the soma and the proximal dendrite. $B$, Detail enlargement from $A$ shows an intermediate and a distal dendrite. The regions of interest are marked white but were shifted to the right to make the dendrite visible. $C$, Voltage-clamp command (bottom trace) and recorded current. $D, \Delta F / F$ corresponding to the current trace in $C$ measured at the four locations depicted in $A$ and $B$. Each $\Delta F / F$ value represents the cumulative signal summated over a time window of $\sim 70$ msec.

LVA current, followed by a step from -45 to $-10 \mathrm{mV}$ to activate the HVA current (Fig. 2C). The corresponding current traces confirm the separate and consecutive activation of the LVA and HVA currents (Fig. 2C). Recordings in which the LVA and HVA currents could not be separated like that were discarded. The LVA-induced fluorescence signal $(\Delta F / F)$ was almost zero at the soma and increased with increasing distance from the soma (Fig. 2D). The HVA-induced fluorescence signal, in contrast, was largest at the soma and declined with an increasing distance from the soma (Fig. 2D). These data indicate an increasing density of LVA currents and a decreasing density of HVA currents with increasing distance from the soma. Statements about current densities along the dendrites are difficult, however, because $\Delta F / F$ might depend on the tapering of the dendrites and the buffer kinetics of the calcium dye. Therefore, we compared separately the LVA current with the HVA current at several dendritic positions. The relative strength of the LVA and HVA currents along the dendrite of one DCN neuron is shown in Figure $3 A$. The LVA and HVA $\Delta F / F$ values were normalized for each position by their sum. The LVA signal increased in comparison to the HVA signal with an increasing distance from the soma indicating a shift in the relative strength of the corresponding barium currents. The same result was obtained when we summarized the response of $11 \mathrm{DCN}$ neurons (Fig. $3 B$ ). The positions in Figure $3 B$ correspond to the soma, a primary dendrite (somatic distance 20-60 $\mu \mathrm{m}$ ), and a secondary dendrite (somatic distance 60-120 $\mu \mathrm{m}$ ). A distance of $100 \mu \mathrm{m}$ corresponds to approximately half the length that is found for dendrites of DCN neurons
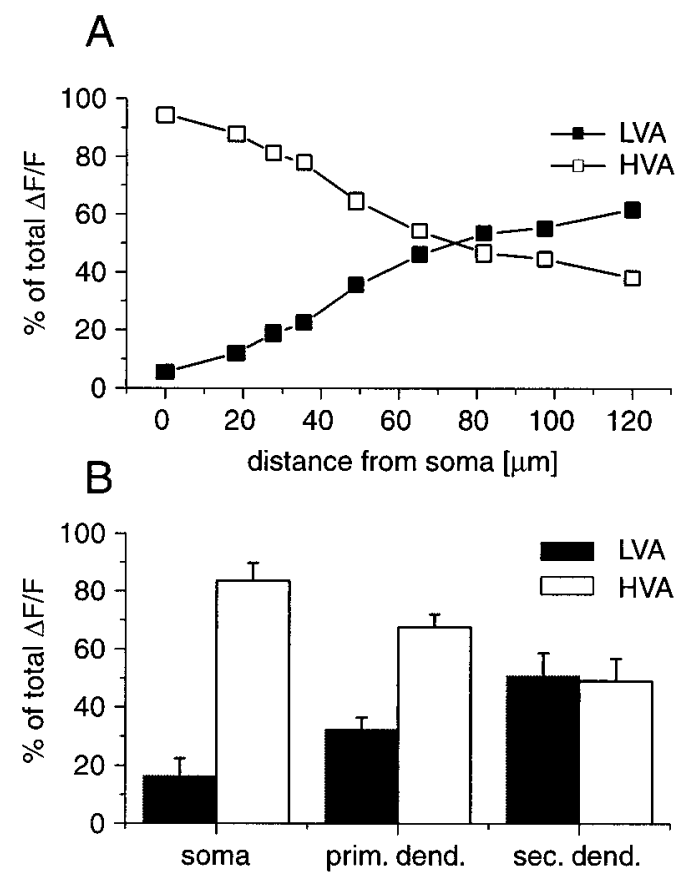

Figure 3. Relative weight of the LVA and HVA currents at several dendritic locations. $A$, Contribution of the LVA and HVA currents from a DCN neuron to the total $\Delta F / F$ in percentage as a function of the distance from the soma. Both values were normalized by their sum for each dendritic position. $B$, The same kind of data for three dendritic positions averaged over $11 \mathrm{DCN}$ neurons (mean $\pm \mathrm{SEM}$ ). The LVA- $\Delta F / F$ values in $A$ and $B$ correspond to the average of the six center values during the LVA voltage command as shown in Figure $2 D$. Accordingly, the HVA $-\Delta F / F$ values correspond to the average of the six center values during the HVA voltage command minus the preceding LVA value.

(Palkovits et al., 1977). Recordings from more distant locations were not possible because of the tapering of the dendrites.

\section{DISCUSSION}

In the present study we examined the spatial distribution of the LVA and HVA currents in DCN neurons by using, for the first time, barium imaging and combining it with voltage clamp. We found that the relative strength between the LVA and HVA currents shifts toward the LVA current with an increasing distance from the soma.

Two potential methodological limitations, i.e., imperfect space clamp and dye saturation, will be considered in the following with respect to their relevance for the described results. An imperfect space clamp can result in a voltage gradient between the soma and the distal dendrites under voltage clamp. This gradient could potentially contribute to the observed shift between the LVA- and HVA-induced $\Delta F / F$ signals. Using the simulation software NEMOSYS, we found a negligible dendritic voltage deviation of $\leq 1 \mathrm{mV}$ for an idealized passive DCN neuron for all voltages that were tested in our experiments (data not shown). Although the activation of barium currents can be expected to result in a larger voltage deviation, our data speak against a severe impairment. Most importantly, we discarded all recordings with signs of imperfect space clamp, such as a broadening of the LVA time course with increasing voltage steps or a prolonged tail current at the end of a voltage step. Therefore, however, we cannot rule out a shift of our cell sample toward neurons with intermediate or low barium current densities. The shift of the surface-volume ratio that goes along with the tapering of dendrites toward their tips 
could potentially result in a decrease of the HVA signal toward the distal dendrites because the preceding LVA current might consume an increasing amount of the available dye-binding sites. Control experiments in which we activated both currents at once showed a linear relationship between the command voltage and the $\Delta F / F$ signal at distal dendrites (data not shown). There, the linear range of the resulting $\Delta F / F$ signal surpassed the total $\Delta F / F$ signal that was elicited by stepping consecutively from -90 to -45 to $-10 \mathrm{mV}$. This is a strong indication against dye saturation. Taken together, it is highly unlikely that imperfect space clamp or dye saturation might account for the observed shift in the relative strength between the LVA and HVA currents toward distal dendrites. Our results therefore indicate that one or both calcium currents might be non-uniformly distributed. Further studies, however, will be necessary to establish the exact calcium channel densities along the dendrites of DCN neurons.

The somatic calcium level of DCN neurons has been shown to depend on their spiking activity (Muri and Knopfel, 1994). DCN neurons are spontaneously active in vitro, and their subthreshold membrane potentials are in a range (Jahnsen, 1986) within which the LVA currents are almost completely inactivated. Therefore, the HVA currents are probably the main source of the spike frequency-related calcium influx. Particular HVA channel types activate calcium-dependent potassium channels in a cell typespecific manner (Marrion and Tavalin, 1998; Pineda et al., 1998). By activating apamin-sensitive, calcium-dependent potassium channels (Aizenman and Linden, 1999), HVA currents are likely to regulate the long-lasting afterhyperpolarization and thereby the overall excitability of DCN neurons. The somatic predominance of the HVA-induced fluorescence signal indicates that the somatic calcium might be of particular importance in this respect. To activate the LVA current, synaptic inputs would first have to hyperpolarize the membrane potential of DCN neurons, and then the LVA current could amplify a subsequent depolarization. On the basis of current injection experiments, it has been suggested that the rebound spiking that DCN neurons show in vitro depends at least in part on the LVA current (Llinás and Muhlethaler, 1988; Aizenman and Linden, 1999). Aizenman and Linden (1999) report that the rebound response was elicited more effectively by the activation of inhibitory synaptic inputs then by somatic current injections. This is consistent with our results of a non-uniform distribution of the LVA current. Approximately $50 \%$ of the inhibitory synapses are located on the distal dendrites of DCN neurons (De Zeeuw and Berrebi, 1995). Therefore, the deinactivation of dendritic LVA channels might be accomplished more effectively by synaptic inputs then by somatic current injections. The predominance of the LVA-induced fluorescence signal in the dendrites of DCN neurons indicates that the LVA current might be particularly important for the integration of dendritic synaptic inputs. A higher density of the LVA current in dendrites has also been found in other neuron types. This has been interpreted to be particularly relevant for the integration of dendritic synaptic inputs (Karst et al., 1993; Christie et al., 1995; Destexhe et al., 1996, 1998; Munsch et al., 1997). A potential amplification of dendritic depolarizations by LVA currents could range from the generation of bursts to more subtle and localized effects. DCN neurons receive a high level of synaptic background activity in vivo (Savio and Tempia, 1985; Stratton et al., 1988) that is likely to have a profound shunting effect (Gauck and Jaeger, 2000). A synaptic shunt in turn would influence the effect of dendritic LVA currents, as a modeling study of thalamic relay neurons has demonstrated (Destexhe et al., 1998). Our results indicate a significant role for the spatial distribution of calcium currents in determining synaptic integration and activity control in DCN neurons.

\section{REFERENCES}

Aizenman CD, Linden DJ (1999) Regulation of the rebound depolarization and spontaneous firing patterns of deep nuclear neurons in slices of rat cerebellum. J Neurophysiol 82:1697-1709.

Aizenman CD, Linden DJ (2000) Rapid, synaptically driven increases in the intrinsic excitability of cerebellar deep nuclear neurons. Nat Neurosci 3:109-111.

Aizenman CD, Manis PB, Linden DJ (1998) Polarity of long-term synaptic gain change is related to postsynaptic spike firing at a cerebellar inhibitory synapse. Neuron 21:827-835.

Artola A, Singer W (1993) Long-term depression of excitatory synaptic transmission and its relationship to long-term potentiation. Trends Neurosci 16:480-487.

Christie BR, Eliot LS, Ito K, Miyakawa H, Johnston D (1995) Different $\mathrm{Ca}^{2+}$ channels in soma and dendrites of hippocampal pyramidal neurons mediate spike-induced $\mathrm{Ca}^{2+}$ influx. J Neurophysiol 73:2553-2557.

Coulter DA, Huguenard JR, Prince DA (1989) Calcium currents in rat thalamocortical relay neurones: kinetic properties of the transient, low-threshold current. J Physiol (Lond) 414:587-604.

Destexhe A, Contreras D, Steriade M, Sejnowski TJ, Huguenard JR (1996) In vivo, in vitro, and computational analysis of dendritic calcium currents in thalamic reticular neurons. J Neurosci 16:169-185.

Destexhe A, Neubig M, Ulrich D, Huguenard JR (1998) Dendritic lowthreshold calcium currents in thalamic relay cells. J Neurosci 18:3574-3588.

De Zeeuw CI, Berrebi AS (1995) Postsynaptic targets of Purkinje cell terminals in the cerebellar and vestibular nuclei of the rat. Eur J Neurosci 7:2322-2333.

Fox AP, Nowycky MC, Tsien RW (1987) Kinetic and pharmacological properties distinguishing three types of calcium currents in chick sensory neurones. J Physiol (Lond) 394:149-172.

Gauck V, Jaeger D (2000) The control of rate and timing of spikes in the deep cerebellar nuclei by inhibition. J Neurosci 20:3006-3016.

Huguenard JR (1996) Low-threshold calcium currents in central nervous system neurons. Annu Rev Physiol 58:329-348.

Jahnsen H (1986) Electrophysiological characteristics of neurons in the guinea-pig deep cerebellar nuclei in vitro. J Physiol (Lond) 372:129-147

Johnston D, Hoffman DA, Magee JC, Poolos NP, Watanabe S, Colbert CM, Migliore M (2000) Dendritic potassium channels in hippocampal pyramidal neurons. J Physiol (Lond) 525:75-81.

Karst H, Joels M, Wadman WJ (1993) Low-threshold calcium current in dendrites of the adult rat hippocampus. Neurosci Lett 164:154-158.

Llinás R, Muhlethaler M (1988) Electrophysiology of guinea-pig cerebellar nuclear cells in the in vitro brain stem-cerebellar preparation. J Physiol (Lond) 404:241-258.

Magee JC (1999b) Dendritic Ih normalizes temporal summation in hippocampal CA1 neurons. Nat Neurosci 2:508-514.

Magee JC (1999a) Voltage-gated ion channels in dendrites. In: Dendrites (Stuart GJ, Spruston N, Haeusser M, eds), pp 139-160. Oxford: Oxford UP.

Marrion NV, Tavalin SJ (1998) Selective activation of $\mathrm{Ca}^{2+}$-activated $\mathrm{K}+$ channels by co-localized $\mathrm{Ca}^{2+}$ channels in hippocampal neurons. Nature 395:900-905.

Munsch T, Budde T, Pape HC (1997) Voltage-activated intracellular calcium transients in thalamic relay cells and interneurons. NeuroReport 8:2411-2418.

Muri R, Knopfel T (1994) Activity induced elevations of intracellular calcium concentration in neurons of the deep cerebellar nuclei. J Neurophysiol (Lond) 71:420-428.

Palkovits M, Mezey E, Hamori J, Szentagothai J (1977) Quantitative histological analysis of the cerebellar nuclei in the cat. I. Numerical data on cells and synapses. Exp Brain Res 28:189-209.

Pineda JC, Waters RS, Foehring RC (1998) Specificity in the interaction of $\mathrm{HVA} \mathrm{Ca}^{2+}$ channel types With $\mathrm{Ca}^{2+}$-dependent AHPs and firing behavior in neocortical pyramidal neurons. J Neurophysiol 79:2522-2534.

Raman IM, Gustafson AE, Padgett D (2000) Ionic currents and spontaneous firing in neurons isolated from the cerebella nuclei. J Neurosci 20:9004-9016.

Savio T, Tempia F (1985) On the Purkinje cell activity increase induced by suppression of inferior olive activity. Exp Brain Res 57:456-463.

Stratton SE, Lorden JF, Mays LE, Oltmans GA (1988) Spontaneous and harmaline-stimulated Purkinje cell activity in rats with a genetic movement disorder. J Neurosci 8:3327-3336. 